\title{
A Novel PS1 Gene Mutation in a Large Aboriginal Kindred
}

\author{
Rachel Butler, B. Lynn Beattie, Umamon Puang Thong, Emily Dwosh, \\ Colleen Guimond, Howard H. Feldman, Ging-Yuek Robin Hsiung, \\ Ekaterina Rogaeva, Peter St. George-Hyslop, A. Dessa Sadovnick
}

\begin{abstract}
Background: There is currently little information on the genetic epidemiology of Alzheimer disease (AD) among North American Aboriginal populations. No cases of familial AD (FAD) in these populations have been published to date. Methods: Here, we describe a large North American Aboriginal kindred with early onset FAD (EOFAD) in which genetic testing was done. Results and Conclusions: A novel Presenilin 1 (PS1) gene mutation (L250F) has been identified. In contrast to the three previously reported families with PS1 codon 250 mutations, affected members of this kindred demonstrate neither myoclonus nor seizures. Furthermore, the identification of a PS1 mutation in a North American Aboriginal kindred presents several unique challenges with respect to knowledge transfer and continuity of care in a geographically remote and culturally distinct community.
\end{abstract}

\begin{abstract}
RÉSUMÉ: Une nouvelle mutation du gène PS1 dans une grande famille autochtone. Contexte : Il existe peu d'informations sur l'épidémiologie génétique de la maladie d'Alzheimer (MA) chez les peuples autochtones de l'Amérique du Nord. Aucun cas de MA familiale (MAF) chez ces populations n'a été publié à ce jour. Méthodes : Nous décrivons une grande famille autochtone nord-américaine atteinte de MAF à début précoce chez qui nous avons effectué un dépistage génétique. Résultats et conclusions : Nous avons identifié une nouvelle mutation (L250F) du gène de la préséniline 1 (PS1). Contrairement aux trois familles porteuses d'une mutation du codon 250 de PS1 rapportées antérieurement, les membres de cette famille qui sont atteints ne présentent pas de myoclonus ou de crises convulsives. De plus, l'identification d'une mutation du gène PS1 dans une famille autochtone nordaméricaine présente plusieurs défis uniques en ce qui concerne le transfert de connaissances et le suivi dans une communauté culturelle différente vivant en région éloignée.
\end{abstract}

Can. J. Neurol. Sci. 2010; 37: 359-364

Alzheimer disease (AD) is the most common adult neurodegenerative condition. Early onset $\mathrm{AD}$ accounts for approximately $6-7 \%$ of all $\mathrm{AD}$, and within this group, $13 \%$ are early onset familial AD (EOFAD) ${ }^{1}$. To date, three genes, each with many mutations, have been identified in EOFAD ${ }^{2}$ : Amyloid $\beta$ precursor protein (APP); Presenilin 1 (PS1); and Presenilin 2 (PS2). Up to $71 \%$ of EOFAD are caused by mutations in one of these three genes ${ }^{1}$.

Mutations of the APP gene located on chromosome 21q21 account for up to $18 \%$ of EOFAD ${ }^{3}$. To date (July 2009), there are 32 proven pathogenic APP mutations (www.molgen.ua.ac.be/ Admutations). The PS2 gene on chromosome 1q31.42 accounts for up to $4 \%$ of $\mathrm{EOFAD}^{3}$, with 14 proven pathogenic mutations identified to date (www.molgen.ua.ac.be/Admutations). By contrast, the PS1 gene on chromosome 14q24.3 accounts for up to $78 \%$ of $\mathrm{EOFAD}^{3}$, with 176 pathogenic mutations confirmed to date (www.molgen.ua.ac.be/Admutations).

There are reported differences in PS1 EOFAD case characteristics at the clinical, neuropsychological, neuroimaging and neuropathological levels ${ }^{4}$. Presenilin 1 mutation carriers demonstrate both inter- and intra-familial heterogeneity with respect to age of onset and clinical/neuropathological phenotype. The reported age of onset ranges from the 20's to age $68^{4}$. While most PS1 mutations show complete penetrance by age 60 , there are some exceptions including mutations A79V; M139V; I143F; H163R, H163Y; A231V; L271V; E273A and possibly C410Y ${ }^{4}$. Symptoms reported to be PS1 mutation-specific include myoclonus, seizures, parkinsonism, extrapyramidal signs,

From the Department of Medical Genetics (RB, ED, CG, ADS), Division of Neurology (UPT, HHF, GYRH, ADS), Faculty of Medicine, Division of Geriatric Medicine (BLB), Faculty of Medicine; UBC Hospital Clinic for Alzheimer Disease \& Related Disorders (RB, BLB, UPT, ED, HHF, GYRH), Vancouver, BC; Department of Medicine and Centre for Research in Neurodegenerative Diseases (ER, PSG), University of Toronto, Ontario, Canada; Neuroscience Bristol-Myers (HHF) Squibb Wallingford, CT, USA.

Received September 30, 2009. Final Revisions Submitted December 14, 2009. Correspondence to: A.D. Dessa Sadovnick, VCHA-UBC Hospital, 2211 Wesbrook Mall, Vancouver, British Columbia, V6T 2B5, Canada. 
apraxia, dystonia, behavioural and psychiatric symptoms, aphasia, visual agnosia, and ataxia, as well as clinical patterns that may overlap with dementia with Lewy bodies or frontotemporal dementia. Atypical neuropathology findings have included accelerated rates of neurofibrillary tangle formation and neuronal loss, as well as a lack of ischemic changes as compared to sporadic AD. Other neuropathological findings associated with PS1 mutations include variations in amyloid angiopathy, the presence of Pick bodies, cortical Lewy bodies and cotton wool plaques.

To date, three EOFAD families have been reported with amino acid substitutions at codon 250 in the PS1 gene ${ }^{5-7}$. One United Kingdom family had a substitution of leucine by serine $(\mathrm{L} 250 \mathrm{~S})^{5}$. Two other families, one from $\mathrm{Japan}^{6}$ and one from Bulgaria $^{7}$, had a substitution of leucine by valine (L250V) at codon 250. Collectively these families included 16 affected individuals. Detailed medical information was available on 11 individuals. The age of onset ranged from 49-56. Of note, myoclonus without seizures was noted in four affected individuals and myoclonus with seizures was noted in another two individuals. Depression, psychiatric disturbance and behavioral disturbances were described in four individuals.

Here we describe an EOFAD family with a novel mutation at codon 250 in the PS1 gene with a leucine to phenylalanine substitution (L250F). Not only is this a novel mutation but it is also, to our knowledge, the first EOFAD mutation reported in a North American Aboriginal kindred (the specific band is not identified for reasons of confidentiality). The clinical symptoms of the affected individuals in this family are presented and contrasted with previously identified codon 250 PS1 mutation carriers.

\section{Materials \& Methods}

The University of British Columbia's (UBC) Hospital Clinic for Alzheimer's Disease and Related Disorders ("UBCHCARD") has routinely recorded and documented detailed family history information for all referred patients since 1984 and there has long been a special interest on atypical and early onset dementia cases ${ }^{8-10}$. All interactions with the family reported here were done with appropriate UBC Clinical Research Board approval and informed consents.

Between 1998 and 2009, nine family members were referred to UBCH-CARD for dementia assessment given the presence of cognitive impairment within the context of a strong family history of dementia (III:4, III:8, III:9, III:14, IV:2, IV:16, IV:18, $\mathrm{V}: 1$ and $\mathrm{V}: 4)$. A condensed pedigree of this family is shown in Figure 1. Six of these individuals had a clinical diagnosis of Probable AD; one had a clinical diagnosis of Possible AD, and two had clinical diagnoses of Mild Cognitive Impairment. As part of the ongoing clinical and genetic follow-up of this kindred, medical records were obtained with consent on two other affected family members (III:1, III:24) indicating clinical diagnoses of Probable AD. Clinical descriptions, restricted to family reports, were available for an additional five family members (II:1, III:3, III:5, III:6, IV:15) with reported symptoms of AD.

Among the nine documented cases of clinically diagnosed Possible/Probable AD in this kindred (III:1, III:8, III:9, III:24, IV:2, IV:16, IV:18, V:1 and V:4), the median age-of-onset is 56 (ranging from 48 to 66), consistent with early onset AD. Given the striking number of documented or reported early onset AD cases and strongly suggestive autosomal dominant inheritance

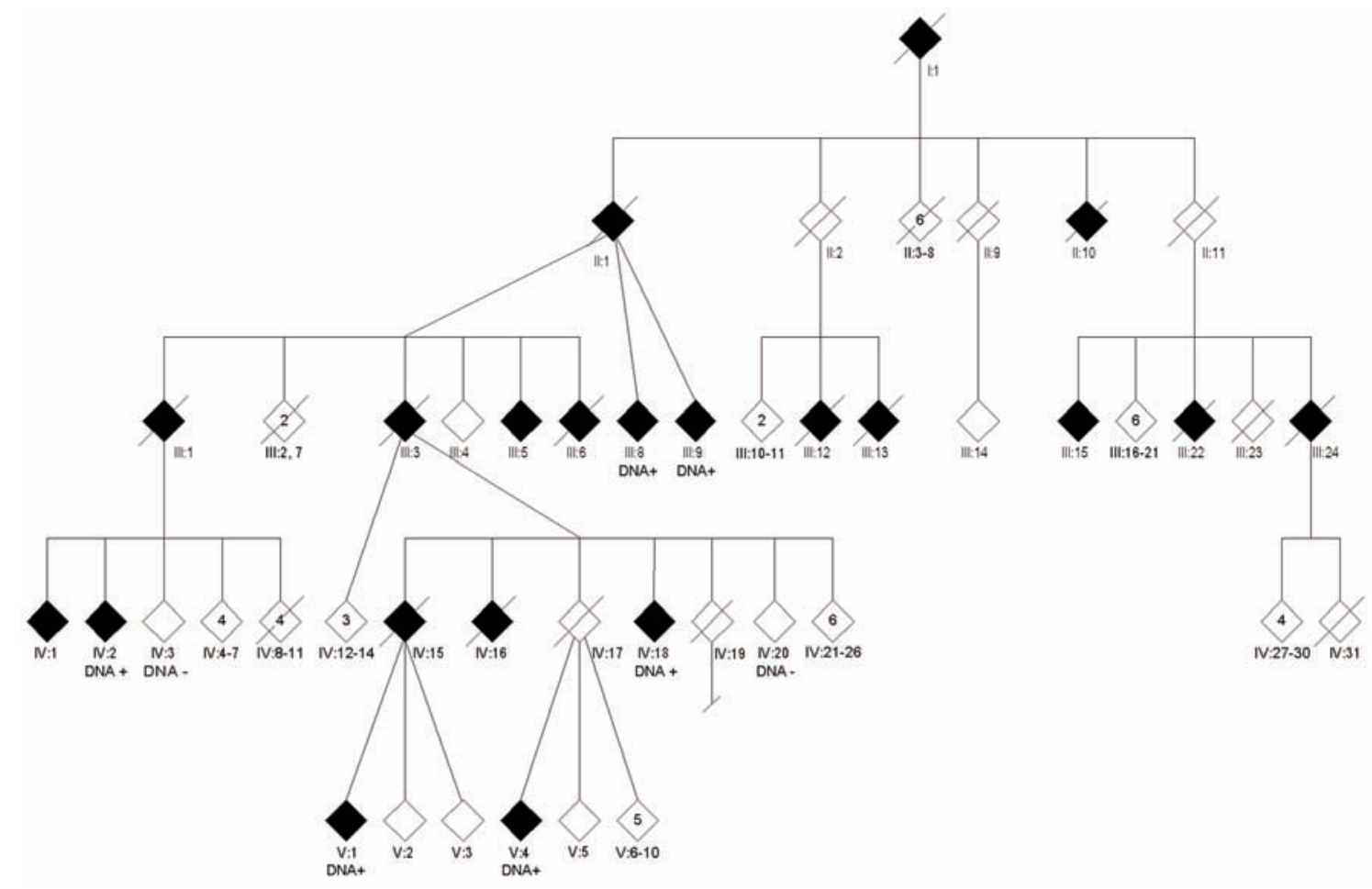

Figure 1: Abridgred and Anonymied family Pedigree. 
pattern in this family, it was suspected that this kindred represented a case of EOFAD.

Genetic testing for EOFAD was offered to several affected family members since 1998 , but V:1 was the first to pursue this option in 2006. Sequencing of exon 16 and 17 of the APP gene and the entire open reading frame of the PS1 gene were done with polymerase chain reactions (PCR) followed by direct DNA sequencing.

\section{RESULTS}

\section{Mutation}

Compared to normal sequences, a $\mathrm{G}>\mathrm{T}$ missense mutation in exon 7 of the PS1 gene (TTG>TTT) at nucleotide position 998, predicted to cause the substitution of leucine at codon 250 by phenylalanine, was identified in V:1. Subsequently, DNA has been obtained from five additional affected family members (III:8, III:9, IV:2, IV:18 and V:4) and two unaffected family members (IV:3, IV:20) who were past the median age of onset in this kindred (56 years). These results confirm that the L250F mutation segregates with the disease in this extended kindred.

\section{FAMiLy OverVIEW}

This family originates from a remote community in British Columbia (BC), Canada, but are now dispersed across the province. To date, nine affected family members have been assessed at the University of British Columbia Hospital Clinic for Alzheimer Disease and Related Disorders; six have been assessed more than once (IV:18 and III:14 had 2 assessments; III:8 had 3; V:1 and IV:2 each had 5; III:9 had 8). Medical records were available regarding two family members with Probable AD (III:1, III:24) and clinical information (through family report) was available on five additional reportedly affected individuals (II:1, III:3, III:5, III:6 and IV:15). Three unaffected adult family members (IV:20, IV:28, IV:30) were seen at $\mathrm{UBCH}-\mathrm{CARD}$ for genetic counseling and baseline cognitive testing. Only one of these individuals pursued the option of genetic testing (IV:20). Of the 16 individuals in this family with documented/reported cognitive impairment, eight are deceased; none had neuropathology. Two living affected family members with PS1 mutations have consented to neuropathology when appropriate.

All of the patients assessed at UBCH-CARD had a normal metabolic screen, which included B12 and thyroid function. No neurological signs were present including focal, motor or sensory loss, cranial nerve problems, change in reflex, gait or balance difficulties. No one had myoclonus or seizures. The majority of the patients had CT head scans, with the exception of IV:18, who also had an MRI.

\section{Family Members Assessed At Ubch-Card \& Genetic Testing Identified Ps1 Mutation:}

For the six individuals with a confirmed PS1 mutation (III:8, III:9, IV:2, IV:18, V:1, V:4), the mean age of onset is 55.7 years. Education ranged from no formal education to a level of Grade 10. The mean rate of decline of all of the individuals in which longitudinal clinical data is available is 0.44 points/year in MMSE, and 3.1 points/year in 3MS (Figure 2A \& 2B). Specific clinical course for these six individuals is as follows:

\section{$\mathrm{V}: 1$}

Education: Grade 10 education.

Previous Medical History: Good general health.

Risk Factors: Head injury from accident and past alcohol abuse and smoking.

Onset Symptoms: Onset began at age 53 when the patient and spouse first noted problems with timing in meal preparation, frequent repetition of stories and social withdrawal. No history of delusions or hallucinations.

1st Assessment at UBC-CARD: First assessed at age 54 and diagnosed with mild AD and placed on Donepezil.

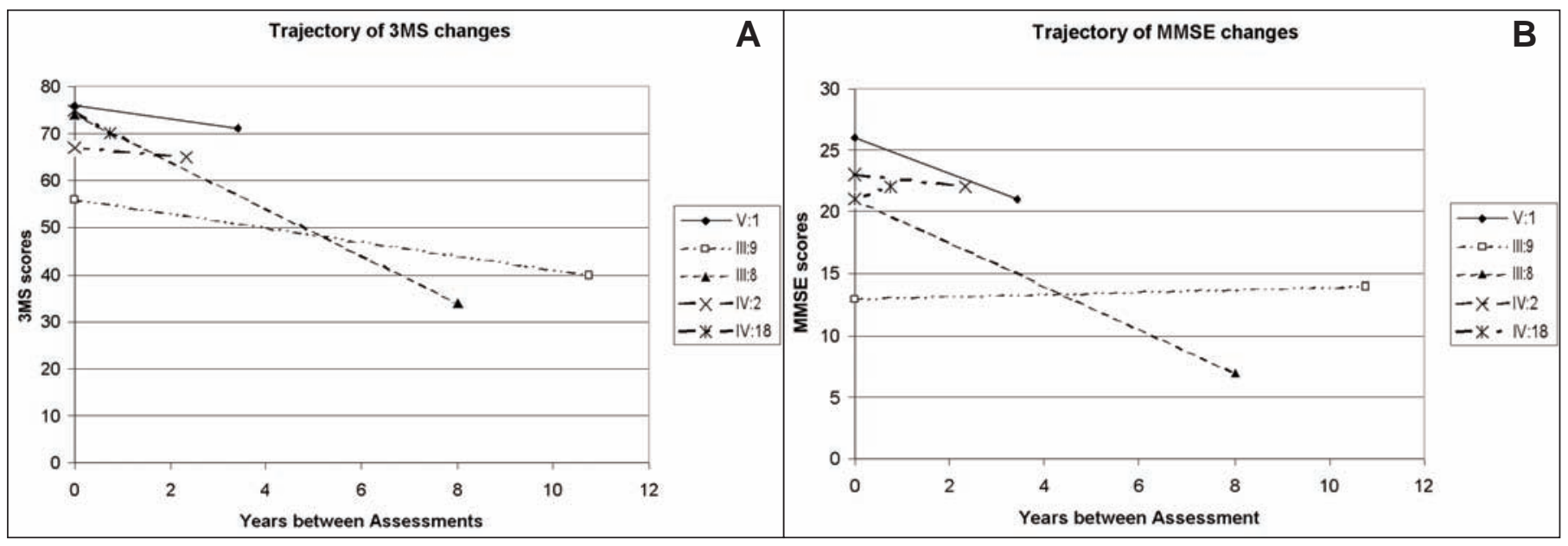

Figure 2: 2A) Trajectory 3MS for patients followed at UBCH-CARD: V:1, III:9,III:8, IV:2, IV:18 (as shown on Figure 1), 2B) Trajectory 3MS for patients followed at UBCH-CARD: V:1, III:9,III:8, IV:2, IV:18 (as shown on Figure 1). 


\section{Clinical Course}

Neuropsychology: Testing at age 56 revealed marked impairment in verbal and visual memory, attention and verbal fluency with milder weaknesses evident in naming abstraction and mental flexibility while her visual spatial abilities, solving skills and attention to visual detail were relatively intact.

Scans: A CT scan demonstrated mild right temporal horn atrophy.

Progress and Current Status: At present, V:1 is 57-years-old and still living with family. V:1 remains independent with personal activities of daily living and function is reasonably stable. V:1 has been on cholinesterase inhibitor for almost three years. No neurological symptoms such as myoclonus or seizures have been noted.

\section{III:8}

Education: Formal education up to Grade 4 or 5.

Previous Medical History: Good general health.

Risk Factors: Depression and past alcohol abuse and smoking.

Onset Symptoms: Reportedly complained of forgetting recent events, conversations and appointments and suffered from depression since age 59.

1st Assessment at UBC-CARD: First assessed at UBCHCARD at age 61. Given a clinical diagnosis of $\mathrm{AD}$ and was placed on Donepezil.

\section{Disease Course}

Neuropsychology: Difficulty in abstraction as well as some mild compromise in retrieval of both verbal and visual information.

Scans: A CT scan demonstrated frontal, parietal and temporal horn atrophy.

Progress and Current Status: By age 69, cognitive function had declined from a moderate to a severe stage. The patient was managed on Donepezil, Memantine and Risperidone. Currently, at age 70 , III- 8 has been placed in a care facility and has illusions and hallucinations. III-8 continues to take a cholinesterase inhibitor and NMDA receptor antagonist as well as small doses of atypical antipsychotic medication. No neurological symptoms such as myoclonus or seizures have been noted.

\section{III:9}

Education: No formal education.

Previous Medical History: Diagnosed with lupus at age 52, which is stable from long-term use of Plaquenil. Long history of depression and anxiety as well as suicidal and paranoid ideations.

Risk Factors: Past alcohol abuse and smoking. Possible head injury(ies) due to abusive spouse.

Onset Symptoms: Complained of memory loss since age 59.

1st Assessment at $\mathbf{U B C H}-\boldsymbol{C A R D}$ : First assessed at age 62.

\section{Disease Course}

Neuropsychology: Decreased attention, decreased verbal recall and poor construction abilities. Clinical picture was compatible with possible $\mathrm{AD}$ with possible confounders being prolonged lupus and depression.

Scans: A CT scan demonstrated frontal, parietal and temporal horn atrophy.
Progress and Current Status: Followed at UBCH-CARD seven more times between ages 62 and 73, showing behavioral changes which included becoming more irritated, upset and anxious in social situations. Multiple attempts were made for inception of Donepezil therapy, starting at age 64 but it was not until age 72 that the patient took it regularly. At age 73, III-9 lives with a spouse in an assisted living care facility. The patient has had a significant decline in instrumental activities of daily living and requires assistance for personal care. III-9 continues to be very anxious and perseverative with symptomatic treatment including antidepressants, which are helpful. No neurological symptoms such as myoclonus or seizures have been noted.

\section{IV:2}

Education: Grade 8 education.

Previous Medical History: Diagnosed and treated for breast cancer at age 49 and Menieres disease from age 57. History of depression associated with bereavement at age 58 .

Risk Factors: Past alcohol abuse and smoking. Possible head injury(ies) due to abusive spouse.

Onset Symptoms: Complained about memory problems at age 56 .

1st Assessment at UBCH-CARD: First assessed at age 59. Particularly had trouble remembering temporal events and was confused with dates, repeated questions, and often misplaced objects. IV-2 was reportedly having more behavioral concerns including possible hallucinations during which she reported seeing other people at night. The patient was diagnosed with mild AD.

\section{Disease Course}

Neuropsychology: Demonstrated difficulty with recall and temporal disorientation.

Scans: A CT scan demonstrated frontal and temporal horn atrophy.

Progress and Current Status: Started taking Galantamine and was followed four more times at UBCH-CARD between age 59 and 62. Currently aged 62, IV-2 was placed in a care facility. The patient began exhibiting more behavior changes and was given an atypical antipsychotic medication for this. IV-2 is reasonably independent in personal care, but requires assistance with most other activities of daily living. To date, there are no neurological symptoms such as myoclonus or seizures.

\section{IV:18}

Education: Grade 10 education.

Previous Medical History: Good general health.

Risk Factors: Past alcohol abuse, smoking and head injury from accident.

Onset Symptoms: Reported a gradual and progressive loss of short-term memory since age 59. Initially seen at age 64 at a local clinic. IV-18 was repetitive and often misplaced items and was given a clinical diagnosis of probable $\mathrm{AD}$ and placed on a combination of Donepezil and Memantine.

1st Assessment at UBCH-CARD: First assessed at age 66. The clinical diagnosis of AD was confirmed.

\section{Disease Course}

Neuropsychology: N/A

Scans: A CT scan demonstrated moderate prominence of the temporal horns. There was no white matter change. 
Progress and Current Status: Currently age 67, the patient's memory has remained fairly stable and he is independent for most activities of daily living. To date, there are no neurological symptoms such as myoclonus or seizures.

\section{$\mathrm{V}: 4$}

Education: Grade 10 equivalent education.

Previous Medical History: Good health other than a hearing problem in one ear as the result of a childhood injury.

Risk Factors: Past alcohol abuse and smoking. Head injury due to an accident.

Onset Symptoms: Reportedly began to have difficulty organizing her work since age 47.

1st Assessment at UBCH-CARD: First assessed at age 52. The neurological exam was normal with the exception of the hearing loss. Given a clinical diagnosis of AD.

\section{Disease Course}

The patient increasingly shows less insight into what is happening. V-4 needs to write everything down and then loses the notes.

Neuropsychology: N/A

Scans: A CT scan demonstrated frontal, parietal and temporal horn atrophy.

Progress and Current Status: Currently age 52, V-4 lives with a spouse and is increasingly unable to make decisions and now hoards items. The patient continues to drive and shop but has problems with homework in an adult education class. To date, there are no neurological symptoms such as myoclonus or seizures.

\section{Iv. Clinical Assessment At UbCh-Card; No Genetic Testing}

III:4, III:14, IV:16, IV:28 and IV:30 were all assessed at UBCH-CARD due to their family history of dementia. IV:16 died at age 70 and no autopsy was performed. III:4's cognitive function has reportedly improved at age 86. III:14 was reevaluated at age 63 and his cognitive testing scores improved. IV:28 and IV:30 were assessed below the mean age of onset in this kindred and did not pursue predictive testing.

\section{Medical Records OnLy (Not Assessed At UbCh-CaRd)}

Medical records were available on III:1 and III:24, which reported they both had $\mathrm{AD}$. Their age of onset ranged from late 50 's to 60's and they died at ages 71 and 64, respectively. Neither individual had neuropathology.

\section{Vi. Information From Family Members (No Medical ReCORds, Not ASSESSED AT UbCh-CARD):}

II:1, III:3, III:5, III:6 and IV:15 all had reported memory concerns (age of onset ranging from 40's to 60's; age of death ranging from 50 to 82 ). Autopsy reports were not performed on any of these individuals and medical records were not available.

\section{Discussion}

The PS1 mutation reported here is both novel (L250F) and, as far as we know, the first reported genetic mutation causative for $\mathrm{AD}$ in a North American Aboriginal kindred. The ability of
UBCH-CARD to follow several members of this kindred longitudinally has allowed for some clinical comparisons between our family with its novel codon 250 mutation and previously documented families with other PS1 codon 250 mutations. To date, clinical phenotype appears to be most consistent with typical AD. Alcoholism is a major confounder in this kindred, with most affected individuals (III:1, III:3, III:8, III:24, IV:2, IV:16, IV:18, V:1 and V:4) having significant exposure. Head injury may also be a potential confounder (III:24, V:1 and V:4). However, it is difficult to assess how much influence, if any, these confounders have on the ages of onset and symptoms. It is possible that these confounders may lead to a younger age of onset and/or hasten the disease progression. Further follow-up and documentation of clinical symptoms may help clarify this in the future. In contrast to the three previously reported families with codon 250 mutations $^{5-7}$, there have been no reports of either myoclonus or seizures in the UBCH-CARD kindred. It remains to be seen if these will develop over time and whether family members will display any atypical neuropathological findings.

As illustrated by Figure 1, although this kindred is very large, relatively few family members have been seen at UBCH-CARD. There are several possible explanations. Literature ${ }^{11}$ suggests that as there are so many more immediate service needs and health care priorities facing Aboriginal communities that cognitive research may not be a priority. The Aboriginal population also may not perceive $\mathrm{AD}$ as a medical $\mathrm{crisis}^{11}$. In Aboriginal culture, the elderly individuals are usually cared for at home and help from outside services is often not sought out. This may be another challenge for early assessment and treatment if individuals are not brought to the attention of the health care system ${ }^{11}$. The vast remoteness of many of the individuals' places of residence makes it difficult to provide outreach and education. It is also been shown that Aboriginal people are more mobile than other Canadians, with one in five Aboriginal people moving within the past year ${ }^{12}$. This creates challenges in providing long-term clinical services and education.

Furthermore, this case illustrates the delicate nature of disseminating genetic findings among a large family that is dispersed across a province. Genetic counseling was done carefully to maintain confidentiality. This raised specific challenges when attempting to document an accurate pedigree and discussing the progress of genetic testing among the family.

Now that a genetic mutation has been identified in this kindred, predictive testing is available to individuals who are below the age of onset and would like to determine if they will get the disease in the future. Following the protocol for Huntington's disease ${ }^{13}$, predictive testing is usually carried out over several appointments to ensure that individuals clearly understand all the social, personal and family implications of such testing. In this kindred, the distance of most individuals' places of residence from UBCH-CARD makes frequent appointments for predictive testing logistically and economically extremely difficult. We are currently strategizing ways to ensure proper education, assessments, genetic counseling and follow-up to help with addressing some of these issues. UBCH-CARD organized a clinic day dedicated to affected and unaffected members of this kindred to share the genetic finding in the family 
and to answer any questions. Three members of UBCH-CARD also went to this remote community to provide education and clinical assessments.

To date, there is very limited information on dementia in general and $\mathrm{AD}$ in particular within Aboriginal populations of Canada and the USA. In fact, the Canadian Study of Health and Aging (CSHA) ${ }^{14}$ specifically excluded Aboriginal people from the study cohort. There appears to be some evidence for AD and/or dementia to be less common in this population ${ }^{11,15-17}$ although longitudinal, population-based studies have yet to be done. Alzheimer disease may very well be under-reported in these populations due to confounders such as alcoholism and vascular disease, as well as limited access to medical services.

In 2006, the Aboriginal population accounted for almost $4 \%$ of Canada's total population with British Columbia being the second most populous province having $4.4 \%$ of its population being Aboriginal ${ }^{12}$. Life expectancy, which can of course censor the population at risk for $\mathrm{AD}$, remains lower in the Aboriginal population compared to other Canadians although this is improving. To illustrate, the number of Aboriginal seniors (aged over 65) has increased $40 \%$ between 1996 and 2001 ${ }^{12}$. Thus, with $\mathrm{AD}$ being the most common adult neurodegenerative condition $^{1}$, it is reasonable to expect an increase in sporadic AD among the Aboriginal population in the near future. Although the kindred reported here is due to a specific PS1 mutation, the issues surrounding the medical and social impact of dementia in our kindred can be applied to the Aboriginal population as a whole.

\section{CONCLUSION}

A North American Aboriginal kindred with a novel PS1 L250F mutation has been described. To our knowledge, this is also the first genetic mutation causative for AD reported in North American Aboriginals. Clinical data is available on 14 individuals with documented or reported diagnoses of AD, six of whom are confirmed PS1 mutation carriers. This has allowed us to describe the phenotype associated with this mutation. Members of this family will continue to be followed at UBCHCARD and we will obtain further information, potentially including neuropathology. Given the fact that sporadic AD is such a common disease, that the Aboriginal population is growing, and that their life expectancy is increasing, the issues we have addressed in this review will likely have broader relevance to the Aboriginal population in coming years.

\section{ACKNOWLedgments}

The authors thank the families affected by this condition and the health care team for their support in aiding us with the communication and dissemination of these findings. We would also like to thank the North Growth Foundation for their financial support for our trip to provide outreach to this community and Talitha Greenwood for constantly updating the family pedigree.

\section{Author Contributions}

All authors contributed extensively to the work presented in this paper. Clinical assessments and diagnoses were done by Drs. Beattie, Feldman and Hsiung. Genetic information for UBCH-
CARD patients and the extended pedigree as well as the collection and banking of DNA and appropriate genetic counseling was done during the study period by Dr. Sadovnick, Rachel Butler, Emily Dwosh, and Colleen Guimond. Drs. St. George- Hyslop and Rogaeva conducted the laboratory analyses which identified the novel mutation and also confirmed its pathogenicity. Umamon Puang Thong conducted an extensive review of the literature. Rachel Butler, Dr. Beattie and Dr. Sadovnick drafted major portions of the manuscript. All the other authors reviewed the manuscript, made editorial comments and approved the final submission.

\section{REFERENCES}

1. Ertekin-Taner N. Genetics of Alzheimer's disease: a centennial review. Neurol Clin. 2007;25:611-67.

2. Brouwers N, Sleegers K, Van Broeckhoven C. Molecular genetics of Alzheimer's disease: an update. Ann Med. 2008;40:562-83.

3. Hsiung GYR, Sadovnick AD. Genetics and dementia: risk factors, diagnosis \& management. Alzheimer Dement. 2007;3:418-27.

4. Larner AJ, Doran M. Clinical phenotypic heterogeneity of Alzheimer's disease associated with mutations of the presenilin1 gene. J Neurol. 2006;253:139-58.

5. Harvey R, Ellison D, Hardy J, Hutton M, Roques PK, Collinge J, et al. Chromosome 14 familial Alzheimer's disease: the clinical and neuropathological characteristics of a family with a leucine -> serine (L250S) substitution at codon 250 of the presenilin 1 gene. J Neurol Neurosurg Psychiatry. 1998;64:44-9.

6. Furuya H, Yasuda M, Terasawa KJ, Tanaka K, Murai H, Kira J, et al. A novel mutation (L250V) in the presenilin 1 gene in a Japanese familial Alzheimer's disease with myoclonus and generalized convulsion. J Neurol Sci. 2003; 209:75-7.

7. Mehrabian S, Traykoy LD, Raycheva MR, Rademakers M, Cruts $\mathrm{M}$, Van Broeckhoven C. Myoclonus, as an atypical phenotype in familial early onset Alzheimer's disease. 26th IEC Proceedings. Epilepsia. 2005;46:283.

8. Sadovnick AD, Irwin ME, Baird PA, Beattie BL. Genetic studies on an Alzheimer clinic population. Genetic Epidemiol. 1989;6: 633-43.

9. Sadovnick AD, Tuokko H, Horton A, Baird PA. Beattie BL. Familial Alzheimer's disease. Can J Neurol Sci. 1988; 5:142-6.

10. Sadovnick AD. Association between Alzheimer's disease and amyotrophic lateral sclerosis? Can J Neurol Sci. 1990;17:352.

11. Griffin-Pierce T, Silverberg N, Connor D, Jim M, Peters J, Kaszniak $\mathrm{A}$, et al. Challenges to the recognition and assessment of Alzheimer's disease in American Indians of the southwestern United States. Alzheim Dement. 2008;4:291-9.

12. Statistics Canada. (2006). First Nations peoples of Canada: A demographic profile, 2006. Available from: http:// www12.statcan.ca/english/census01/Products/Analyic/ companion/abor/canada.cfm.

13. Craufurd D, Tyler A. Predictive testing for Huntington's disease: protocol of the UK Huntington's Prediction Consortium. J Med Genet. 1992;29;915-18.14. Canadian Study of Health and Aging: study methods and prevalence of dementia. Can Med Assoc J. 1994;150:899-913.

15. Hendrie HC, Hall KS, Pillay N, Rodgers D, Prince C, Norton J, et al. Alzheimer's disease is rare in Cree. Int Psychogeriatr. 1993;5:5-14.

16. Rosenberg RN, Richter RW, Risser RC, Taubman K, Prado-Farmer I, Ebalo E, et al. Genetic factors for the development of Alzheimer disease in the Cherokee Indian. Arch Neurol. 1996; 54:997-1000.

17. Weiner MF, Rosenberg RN, Svetlik D, Hynan LS, Womack KB, White $\mathrm{C}$ 3rd, et al. Comparison of Alzheimer's disease in Native Americans and Whites. Int Pyschogeriatr. 2003;15:367-75. 